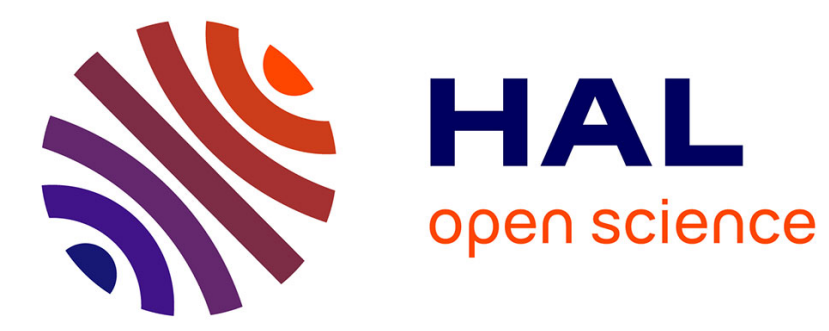

\title{
Dry granular flows: Rheological measurements of the $\mu(\mathbf{I})$-rheology
}

Abdoulaye Fall, Guillaume Ovarlez, David Hautemayou, Cédric Mézière, Jean-Noël Roux, François Chevoir

\section{To cite this version:}

Abdoulaye Fall, Guillaume Ovarlez, David Hautemayou, Cédric Mézière, Jean-Noël Roux, et al.. Dry granular flows: Rheological measurements of the $\mu(\mathrm{I})$-rheology. Journal of Rheology, 2015, 59 (4), pp.1065. 10.1122/1.4922653 . hal-01165682

\section{HAL Id: hal-01165682 \\ https://hal-enpc.archives-ouvertes.fr/hal-01165682}

Submitted on 27 Jun 2019

HAL is a multi-disciplinary open access archive for the deposit and dissemination of scientific research documents, whether they are published or not. The documents may come from teaching and research institutions in France or abroad, or from public or private research centers.
L'archive ouverte pluridisciplinaire $\mathbf{H A L}$, est destinée au dépôt et à la diffusion de documents scientifiques de niveau recherche, publiés ou non, émanant des établissements d'enseignement et de recherche français ou étrangers, des laboratoires publics ou privés. 


\section{Dry granular flows: rheological measurements of the $\mu(I)$-rheology}

A. Fall ${ }^{1}$, G. Ovarlez ${ }^{1,2}$, D. Hautemayou ${ }^{1}$, C. Mézière ${ }^{1}$, J.-N. Roux ${ }^{1}$ and F. Chevoir ${ }^{1}$

${ }^{1}$ Université Paris Est, Laboratoire Navier (CNRS, IFSTTAR, Ecole des Ponts ParisTech), Champs-sur-Marne, France

${ }^{2}$ CNRS, LOF, UMR 5258, F-33600 Pessac, France

\section{Synopsis}

Granular materials do not always flow homogeneously like fluids when submitted to external stress, but often form rigid regions that are separated by narrow shear bands where the material yields and flows. This shear localization impacts their apparent rheology, which makes it difficult to infer a constitutive behaviour from conventional rheometric measurements. Moreover, they present a dilatant behaviour, which makes their study in classical fixed-volume geometries difficult. These features led numerous groups to perform extensive studies with inclined plane flows, which were of crucial importance for the development and the validation of the $\mu(I)$-rheology. Our aim is to develop a method to characterize granular materials with rheometrical tools. Using rheometry measurements in an annular shear cell, dense granular flows of $0.5 \mathrm{~mm}$ spherical and monodisperse beads are studied. A focus is placed on the comparison between the present results and the $\mu(I)$ - rheology. From steady state measurements of the torque and the gap under imposed shear rate $\dot{\gamma}$ and normal force $F_{N}$, we define an inertial number $I$. We show that, at low $I$ (small $\dot{\gamma}$ and/or large $F_{N}$ ), the flow goes to a quasi-static limit, and the response in terms of dimensionless stress or internal friction coefficient $-\mu-$ and solid concentration $-\phi-$ profiles is independent of the inertial number. Upon increasing $I$ (large $\dot{\gamma}$ and/or small $F_{N}$ ), dilation occurs and $\phi$ decreases while $\mu$ increases. The observed variations are in good agreement with previous observations of the literature (Jop et al. 2006; Hatano 2007). These results show that the constitutive equations $\mu(I)$ and $\phi(I)$ of granular materials can be measured with a rheometer. 


\section{Introduction}

Granular matter shows both solid and fluid behavior. Of interest in many industrial processes and in geophysics, granular flows are the focus of very active researches (Duran 2000). These materials are very sensitive to various parameters: geometry of the flow, wall roughness, flow rate, shape and size distribution of the grains, and coupling with the interstitial fluid (Andreotti et al. 2013). Due to their macroscopic size, the interactions between the grains are dissipative (friction and inelastic collisions); the energy lost is then transferred to internal degrees of freedom. The lack of Brownian motion and the dissipative interactions, make the granular material an intrinsically nonequilibrium system.

In the dry case - without interstitial fluid -, the rheology is solely governed by momentum transfer and energy dissipation occurring in direct contacts between grains and with the walls. Despite the seeming simplicity of the system, the behavior of dry granular material is very rich and extends from solid to gaseous properties depending on the flow regime. In the absence of a unified framework, granular flows are generally divided into three different regimes. (i) At low shear, particles stay in contact and interact frictionally with their neighbours over long periods of time. This "quasi-static" regime of granular flow has been classically studied using modified plasticity models based on a Coulomb friction criterion (Schofield \& Wroth 1968; Becker and Lippmann 1977). The response in terms of velocity or solid fraction profiles is independent of the shear rate (Roux \& Combe 2002; GDR Midi 2004). Consequently, if the material remains homogeneous, this state only depends on geometric data (shape and size distribution of the grains) and on the inter-granular friction coefficient. (ii) Upon increasing the deformation rate, a viscous-like regime occurs and the material flows more as a liquid (Forterre \& Pouliquen 2008). In this intermediate regime, the particles experience multi-contact interactions. (iii) At very high velocity, a transition occurs towards a gaseous regime, in which the particles interact through binary collisions (Goldhirsch 2003; Jenkins and Savage 1983).

For the modelling of dense granular flows, the concept of inertial number $I$ has been widely used and investigated with regard to its relationship with dynamic parameters, such as velocity, stress, and friction coefficient which leads to constitutive relations for granular flows. Thus, 'dynamic dilatancy' law and 'friction' law were deduced from discrete simulation of two dimensional simple shear of a granular material without gravity (da Cruz et al. 2005). Those results establish that the flow regime and rheological parameters depend on a single dimensionless number that represents the relative strength of inertia forces with respect 
to the confining pressure, or the combined effect of pressure and shear rate. Indeed, for a three-dimensional granular medium made up of monodisperse spheres ( $d, \rho$ resp. the particle's diameter and density) undergoing simple shear flow at a shear rate $\dot{\gamma}$ under an applied normal confining stress $\sigma$, this dimensionless number - inertial number - is defined as $I=\dot{\gamma} d / \sqrt{\sigma / \rho}$. Alternatively, one may use the Savage number which is the square of the inertial number (Savage \& Hutter 1989) or the Coulomb number (Ancey et al. 1999, see also GDR Midi 2004; Baran \& Kondic 2006; Hatano 2007; Luding 2008). Dimensionless number $I$ can also be seen as the ratio of $d / \sqrt{\sigma / \rho}$, the time scale for grains to rearrange due to the confining stress $\sigma$, to the time scale $\dot{\gamma}^{-1}$ for deformation by the flow. Hence, it characterizes the local "rapidity" of the flow. Thus it was observed that both dimensionless quantities: the internal friction coefficient $\mu=\tau / \sigma$ and the solid fraction $\phi$ are functions of $I$ (GDR Midi 2004; da Cruz et al. 2005; Hatano 2007). Thereby, the inertial number I opened a new path unifying, in a single phenomenological law, many experimental and numerical data in a wide variety of transient flows from the rotating drum to inclined plane flows, where large flowing zones form. A local relation between an apparent friction coefficient and $I$ then successfully captures many aspects of these rapid granular flows (Savage \& Hutter 1989; GDR Midi 2004; Jop et al. 2006; Forterre \& Pouliquen 2008).

Following general results from simulations of planar shear (da Cruz et al. 2005; Iordanoff \& Khonsari 2004), and successful applications to inclined plane flows (Pouliquen \& Forterre 2002; Silbert et al. 2003), the experiments of Jop and co-workers (Jop et al. 2006) were carried out to quantify, for glass beads, the $\mu(I)$ - rheology from the quasi-static to the rapid flow regime, corresponding to moderate inertial number (from 0.01 to 0.5 ) as:

$$
\mu=\mu_{s}+\left(\mu_{2}-\mu_{s}\right) /\left(1+I_{0} / I\right)
$$

in which $\mu_{s}, \mu_{2}$ and $I_{0}$ are three fitting parameters dependent on material properties. According to this law, the internal friction coefficient $\mu$ goes from a minimum value $\mu_{s}$ for very low $I$ up to an asymptotic value $\mu_{2}$ when $I$ increases. The asymptotic value of $\mu$ at high inertial number was not obtained by da Cruz and co-workers who observed an approximately linear increase of the internal friction coefficient from the static internal friction value:

$$
\mu=\mu_{s}+a I
$$


where $\mu_{s}$ and $a$ are two fitting parameters which depend on material properties. In 3Dsimulation studies, Hatano did not either observe the asymptotic value of $\mu$ at high $I$ : from $I=10^{-4}$ to $I=0.2$, he reported a law in which the friction coefficient increases as a power of the inertial number (Hatano 2007):

$$
\mu=\mu_{s}+a I^{n}
$$

It should be pointed out however that this rheology agrees with earlier scaling relations stemming back to Bagnold (Bagnold 1954). Bagnold described a mechanism of momentum transfer between particles in adjacent layers that assumes instantaneous binary collisions between the particles during the flow. Under this assumption, the inverse strain rate is the only relevant time scale in the problem leading, for a constant solid fraction $\phi$, to constitutive relations between the shear stress $\tau$ (resp. normal stress $\sigma$ ) and shear rate $\dot{\gamma}$ of the form $\tau=\rho d^{2} f_{1}(\phi) \dot{\gamma}^{2}$ (resp. $\sigma=\rho d^{2} f_{2}(\phi) \dot{\gamma}^{2}$ ) where $f_{1}$ and $f_{2}$ are functions of the solid fraction $\phi$ only. Bagnold's scaling has been verified for dry grains in both collisional and dense flow regimes (Jenkins \& Savage 1983; Lois et al. 1987; Silbert et al. 2001; Lois et al. 2005; da Cruz et al. 2005). The main difference between the Bagnold and $\mu(I)$ approaches is that: in the first case, $\phi$ is kept constant for one given flow, whereas it varies freely and depends on the flow in the second case. Indeed, if the pressure is controlled, the solid fraction $\phi$ is free to adapt in the system with the evolution of other parameters. If $\phi$ is fixed however and the normal pressure measured, this will fix the value of $I$. Then when $\phi$ is fixed, the expression of $I$ shows that the normal pressure should scale with the square of the shear rate as it was shown experimentally in annular parallel-plate shear cell (Savage \& Sayed 1984).

The applicability of the $\mu(I)$ - rheology has been examined by various studies (Jop et al. 2005, 2006; Hatano 2007; Forterre \& Pouliquen 2008; Ruck et al. 2008; Aranson et al. 2008; Peyneau \& Roux 2008; Staron et al. 2010; Gaume et al. 2011; Tripathi \& Khakhar 2011; Chialvo et al. 2012; Azéma \& Radjai, 2014; Gray \& Edwards 2014; Edwards \& Gray 2015) and many simulations and experiments have shown that the rheology is valid for various flow configurations for different choices of materials, although deviations from this rheology may take place for very slow (quasi-static) flows with small values of inertial number (Aranson et al. 2008; Staron et al. 2010; Gaume et al. 2011). Up to now, there is however in the literature, no experimental data from conventional rheology (rheometer with conventional geometries) describing the $\mu(I)$-rheology of a dry granular material. Indeed, Couette flows of granular 
materials are characterized by the formation of well-defined shear bands that resemble qualitatively the behavior of a yield stress fluid, and make the interpretation of rheometric data tricky. For slow flows, shear banding is generally very strong, with shear bands having a typical thickness of five to ten grain diameters. In this regime of slow flow, the averaged stresses and flow profiles become essentially independent of the flow rate, so that constitutive relations based on relating stresses and strain rates are unlikely to capture the full physics (Schofield \& Wroth 1968; Nedderman 1992; Fenistein \& van Hecke 2003; Fenistein et al. 2004). Moreover, due to the dilatant behaviour of granular materials, constant solid fraction $\phi$ experiments classically made with a Couette shear-cell rheometer are much more difficult to perform than constant friction coefficient $\mu$ experiments such as inclined plane flows. When a granular matter is sheared, the spatial distribution of the shear rate is not always homogeneous. Often, shear is localized near the system boundaries with a shear localization width amounting to a few particle diameters. Nevertheless, depending on the boundary conditions, confining pressure and shear velocity, the bulk of the granular system may exhibit different behaviours. For high shear velocities and small confining pressures - high $I$-, the granular matter flows homogeneously (Koval et al. 2009). However, at small inertial number, it was indeed shown that in confined annular flow at small shear velocities and high confining pressures - small $I$-, the shear may be not homogeneous and solid and fluid phases coexist (Aharonov \& Sparks 2002; Jalali et al. 2002).

In the present work, we show that it is not necessary to develop specific set-ups (such as the inclined plane) to study dense granular flows. Indeed, we show that a simple annular shear cell (Carr and Walker 1968, Savage and Sayed 1984) can be adapted to a standard rheometer to study the rheology of granular materials under controlled confining pressure. It allows us in particular to obtain the dilatancy law $\phi(I)$ and also to study very accurately the quasi-static limit. Thus, from the steady state measurements of the torque and the gap during an imposed shear flow under an applied normal confining stress $\sigma$, we report two laws in which the internal friction coefficient and the solid fraction are functions of a single dimensionless number: the inertial number $I$. An effort is then made to compare the present results to the $\mu(I)$ - rheology described in the literature. Indeed, we show that at low inertial number $I$ (small $\dot{\gamma}$ and/or large $\sigma$ ), the flow goes to the quasi-static limit, and the response in terms of internal friction coefficient $\mu$ and solid fraction $\phi$ profiles is independent of the inertial number. Upon increasing $I$ (large $\dot{\gamma}$ and/or small $\sigma$ ), $\phi$ decreases while $\mu$ increases. The 
observed variations are in good agreement with previous observations of the literature. Importantly, we also show that changing the initial gap size does not significantly affect these results. This suggests that shear localization is mostly absent in the intermediate dense flow regime, although it may still occur when the granular material is slowly sheared (in the quasistatic regime).

\section{Materials and methods}

To investigate the steady flows of dry granular materials and determine the $\mu(I)$ - rheology, three main features are required: (i) to avoid shear banding, (ii) to apply a confining stress in the velocity gradient direction, and (iii) to allow volume fraction variations. If one wants to use a rheometer, (i) implies that the use of a Couette cell should be avoided since it is characterized by a shear stress inhomogeneity that naturally leads to shear-banding. Both the cone-and-plate and the parallel-plate geometries allow a normal force to be imposed in the velocity gradient direction; however, the analysis of the cone-and-plate flow can be performed only at a single gap value, i.e. it cannot be used to characterize a material whose the volume varies under shear. On the other hand, any gap variation in parallel-plate geometry can be accounted for in the determination of the shear rate value. Moreover, shear banding should in principle be avoided in this last geometry since the shear stress is independent of the vertical position in the gap (Macosko 1993). Nevertheless, the shear rate varies along the radial position and is equal to zero in the center. One should thus try to avoid the use of the central zone of the gap. Finally, the material should be confined by lateral walls to make sure that any gap variation actually leads to a material volume fraction variation. These requirements led us to develop a home made annular shear cell, inspired by Boyer et al. 2011, in which pressureimposed measurements can be performed as shown in Figure 1. Annular shear cells have been extensively used to characterize the flow of pharmaceutical powders and dry granular materials (Carr and Walker 1968, Savage and Sayed 1984, Schulze 1996; Schwedes 2003).

We use a granular material made of rigid polystyrene beads (from Dynoseeds) of density $\rho=1050 \mathrm{~kg} / \mathrm{m}^{3}$, of diameter $d=0.5 \mathrm{~mm}$ (with a standard deviation of $5 \%$ ). Spherical beads fill the annular box between two static concentric cylinders with respectively an inner and outer radii of $R_{i}=21 \mathrm{~mm}$ and $R_{o}=45 \mathrm{~mm}$. The width of the annular trough is about $48 \mathrm{~d}$ leading to a ratio of inner to outer wall radii of 0.46 . We also used another annular channel with the same width but with a larger ratio of inner to outer cylinder radii equal to 0.61 ( $R_{i}=38 \mathrm{~mm}$ and $R_{o}=62 \mathrm{~mm}$ ). We have verified that changing the ratio of inner to outer 
193

194

195

196

197

198

199

200

201

202

cylinder radii of the annular shear cell does not significantly affect the results. The filling height (initial gap, $h_{0}$ ) of the annular box is adjustable from a few grain diameters (typically $5 d)$ to $30 d$. The cylinders were finished as smoothly as possible to permit the granular material to slip there as readily as possible. For that, they are made of polyoxymethylene (POM) resin which exhibits a low friction coefficient due to the flexibility of the linear molecular chains. We have measured the friction coefficient at the wall $\mu_{w}$ between polystyrene beads and a plane made of POM by measuring with our rheometer the sliding stress under different normal stresses such as in Jenike’s shear tester (Jenike 1964; Schwedes 2003); it is found to be very small: $\mu_{w} \approx 0.05$.

(a)
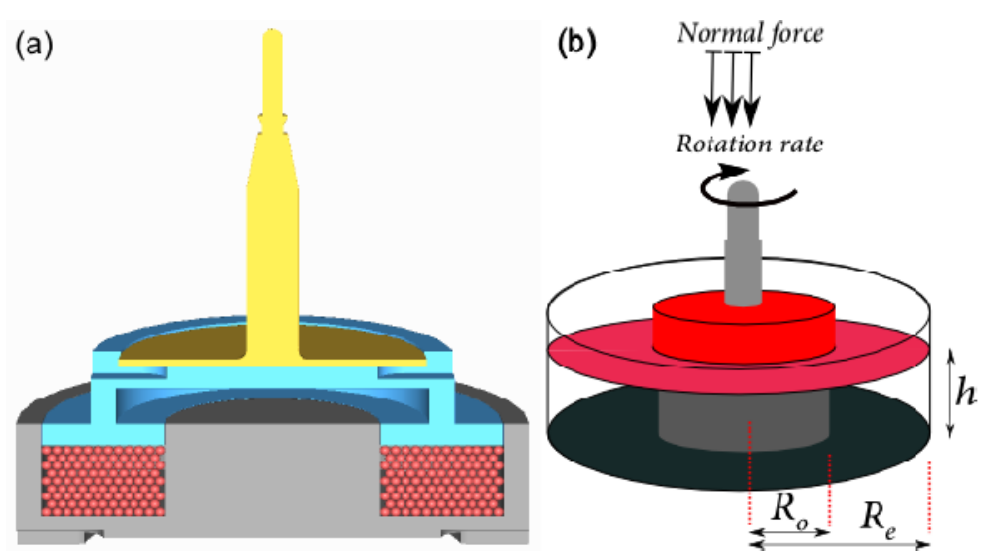

Rain-filling

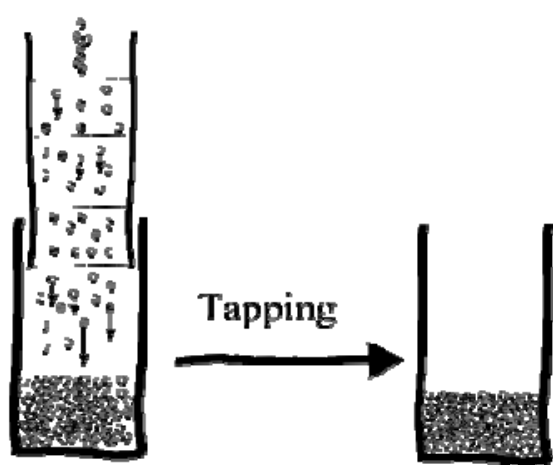

204

Figure 1: (a-b) Cross section of the annular plane shear flow. The shear and pressure are provided by a ring which is assembled on a Kinexus rheometer by Malvern that is free to move vertically while maintaining a constant rotation rate or shear rate and imposed pressure. (c) Rain-filling coupled to tapping to get the denser piling sample ('D').

The experiments were performed initially on a very dense piling $\phi_{0} \approx 0.625$, close to the so called random close packing of 0.637 (Torquato et al. 2000; Camenen et al. 2012) obtained by combining a rain-filling and tapping the box (Ovarlez et al. 2003) to get a reasonably uniform packing (Figure 1b). However, we will show below that the steady state obtained when the material is sheared is the same for an initially looser piling (Figure 2c). Granular beads are then driven by the ring-shaped upper boundary made of PMMA, which is assembled on a Kinexus Pro rheometer by Malvern. To avoid wall slip, both the moving upper boundary and the static lower boundary are serrated, with $0.5 \mathrm{~mm}$ ridges which correspond to the size of grains (Shojaaee et al. 2012). 
220 In our rheometer, instead of setting the value of the gap size for a given experiment, as in

221 previous studies (Schulze 1996; Schwedes 2003) and generally in rheometric measurements, 222 we impose the normal force (i.e. the confining normal stress) and then, under shear, we let the 223 gap size vary in order to maintain the desired value of the normal force. We then have access 224 to instantaneous measurements of the driving torque $T$ and the gap $h$ for imposed normal 225 force $F_{N}$ and shear rate $\dot{\gamma}$ : in this case, the solid fraction $\phi$ is not fixed, but adjusts to the 226 imposed shear. However, it remains important to notice that, in order to keep the imposed 227 shear rate constant, the rheometer adjusts the rotation velocity $\Omega$ since the gap varies as will 228 be discussed below.

230 A typical measurement is shown in Figure 2, where we start out with a given gap $h_{0} \approx 6 d$, 231 impose a constant shear rate $\dot{\gamma}$ and normal force $F_{N}$ and measure the torque $T$ and the gap $232 h$ as a function of strain (or time). The system reaches a steady state after a certain amount of 233 shear strain but we carefully compare the transient dynamics of these quantities, beginning 234 with freshly poured grains for two preparations, 'rain piling' and 'rain coupled to tapping 235 piling' (resp.) which allow us to get the looser ('L') piling and the denser ('D') piling (resp.) 236 samples with an initial solid fraction of 0.612 and 0.625 (resp.).

237 Let us start with the imposed quantities. In both cases, a small variation of the rotation 238 velocity is seen (Figure 2a). As discussed above, this variation is a signature of the shear239 induced gap variations; it ensures that the shear rate is constant at any time or strain (see 240 inset). We also see that, in both cases, the normal force reaches quickly the stationary targeted 241 value and remains steady during the whole experiment (Figure 2b). Nevertheless, the short 242 transient behavior is different: in the looser sample (' $L$ '), a significant decrease of the normal 243 force is initially observed while a very sharp increase is observed in the densest piling (' $D$ '). 244 The reason is that the densest system initially wants to dilate, and the rheometer response is 245 not fast enough to allow for this fast dilation at constant normal force. By contrast, the loose 246 sample initially wants to compact, and again the rheometer response is not fast enough to 247 allow for this fast compaction at constant normal force. 

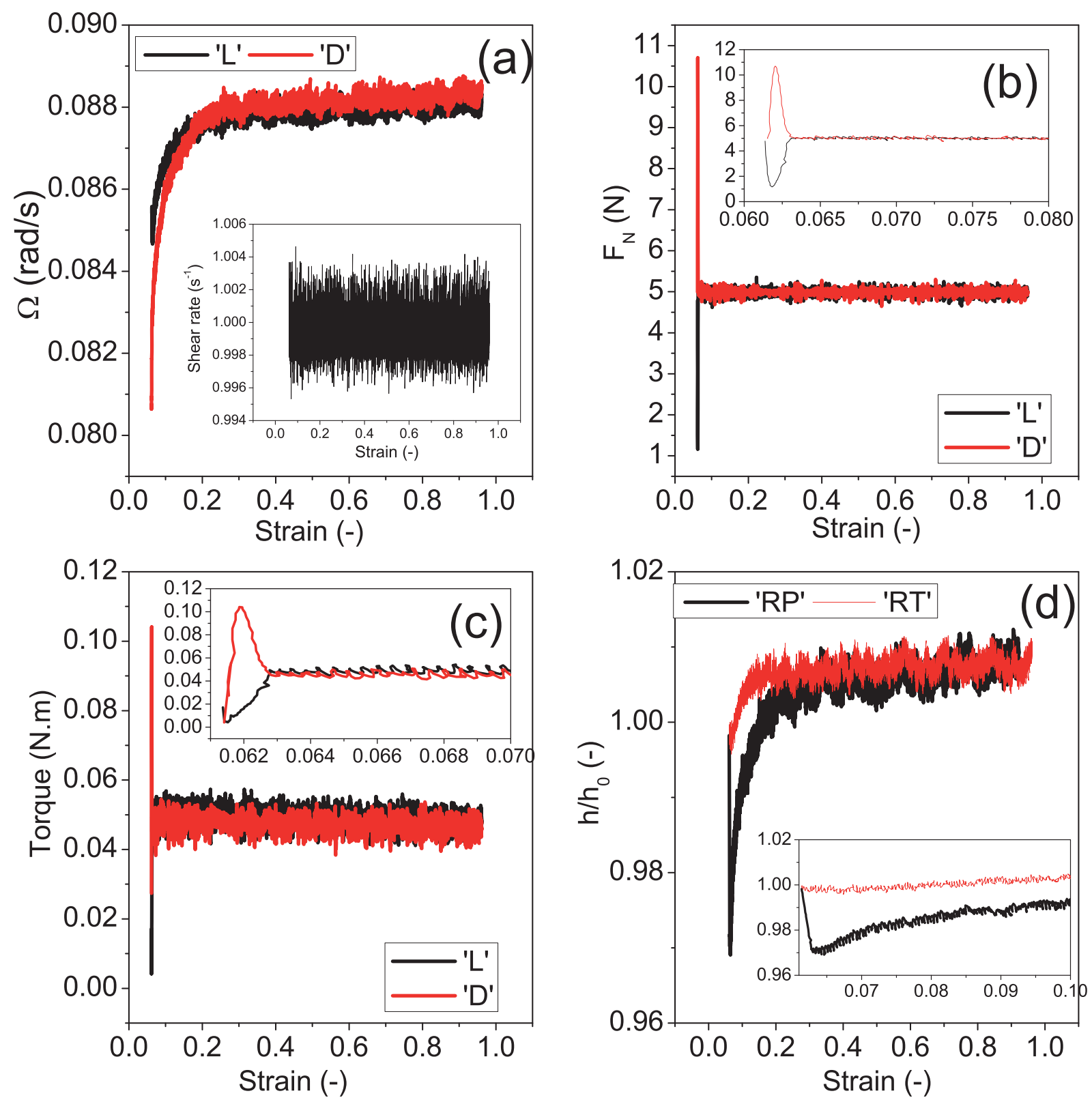

250 Figure 2: Comparison between samples from the two different pilings: rain piling (which 251 allows us to get the looser piling sample ' $\mathrm{L}$ ') and rain piling coupled to tapping (that lets us to 252 get the denser piling sample ' $D$ ') of the (a) rotational rate against strain; Inset: the imposed 253 shear rate vs. strain, (b) imposed normal force as a function of strain, (c) driving torque and 254 (d) gap size rescaled by the initial gap against strain. The insets in (b-c-d) are the same data 255 showing the sharp increase/decrease of the normal force, the overshoot of the torque and the 256 gap change at the beginning of shearing.

258 The transient dynamics of the measured quantities (torque and gap size) also shows a clear 259 difference between the two samples. In Figure 2c, we see that, in the loosest piling (' $L$ '), the torque increases before reaching the steady state regime. By contrast, there is an overshoot 
261

262

263

264

265

266

267

268

269

270

271

272

273

274

275

276

277

278

279

280

281

282

283

284

285

286

287

288

289

290

291

within the densest sample ('D'), followed by a fast decrease of the torque: the peak strongly depends on the imposed normal force and/or on the shear rate; the decrease corresponds to the continuous dilation of the material under shear. Indeed, in the same time, the gap is not fixed, but adjusts to the imposed shear (Figure 2d). Shear dilation is observed: the measured gap increases up to a constant value since the average of the initial solid fraction is very close to the random close packing. In this regime, shearing necessarily implies immediate dilatancy of the granular media. In contrast, an initially looser sample first compact instead of dilate under shear: when the system is driven under a fixed normal pressure, the granular packing undergoes compaction before dilation into the final state once sufficiently compacted (Wroth 1958).

The most important observation is that the same steady state is reached (same torque and same solid fraction) for the two initial states. This indicates that the material history has been erased, and that we are actually studying the material steady response as a function of $\dot{\gamma}$ and $F_{N}$ only. As a consequence, there is no need for the sample preparation for the grains we study, namely monodisperse spheres (note that this might not be a general result). All the annular shear cell requires is that the sample is sheared at the required normal pressure until the critical state is reached. However it is necessary to adopt a reference packing state and ensure that the piling method used allows us to achieve sufficient repeatability for a given set materials and system parameters. Hence, we prepare all samples in the same way: a very dense material, from a rain piling coupled to tapping, is used in all the experiments presented below (the initial average volume fraction is 0.625 ).

\section{Experimental results and analyses}

We have measured the driving torque and the gap as a function of shear strain for various imposed normal force $F_{N}$ (between 1 and and $5 N$ ) and applied shear rate $\dot{\gamma}$ (between 0.01 and $77 \mathrm{~s}^{-1}$ ) for a given gap $h_{0} \approx 6 d$.

In Figure $3(\mathrm{a}, \mathrm{b})$, we show those measurements for $F_{N}=3 N$ and various $\dot{\gamma}$. At low shear rate, the driving torque increases slowly before reaching a steady plateau within strain of order of unity. Meanwhile, the gap fluctuates around its initial value (we show the gap size rescaled by its initial value before shearing $h / h_{0}$ called the rescaled gap in the following.). 

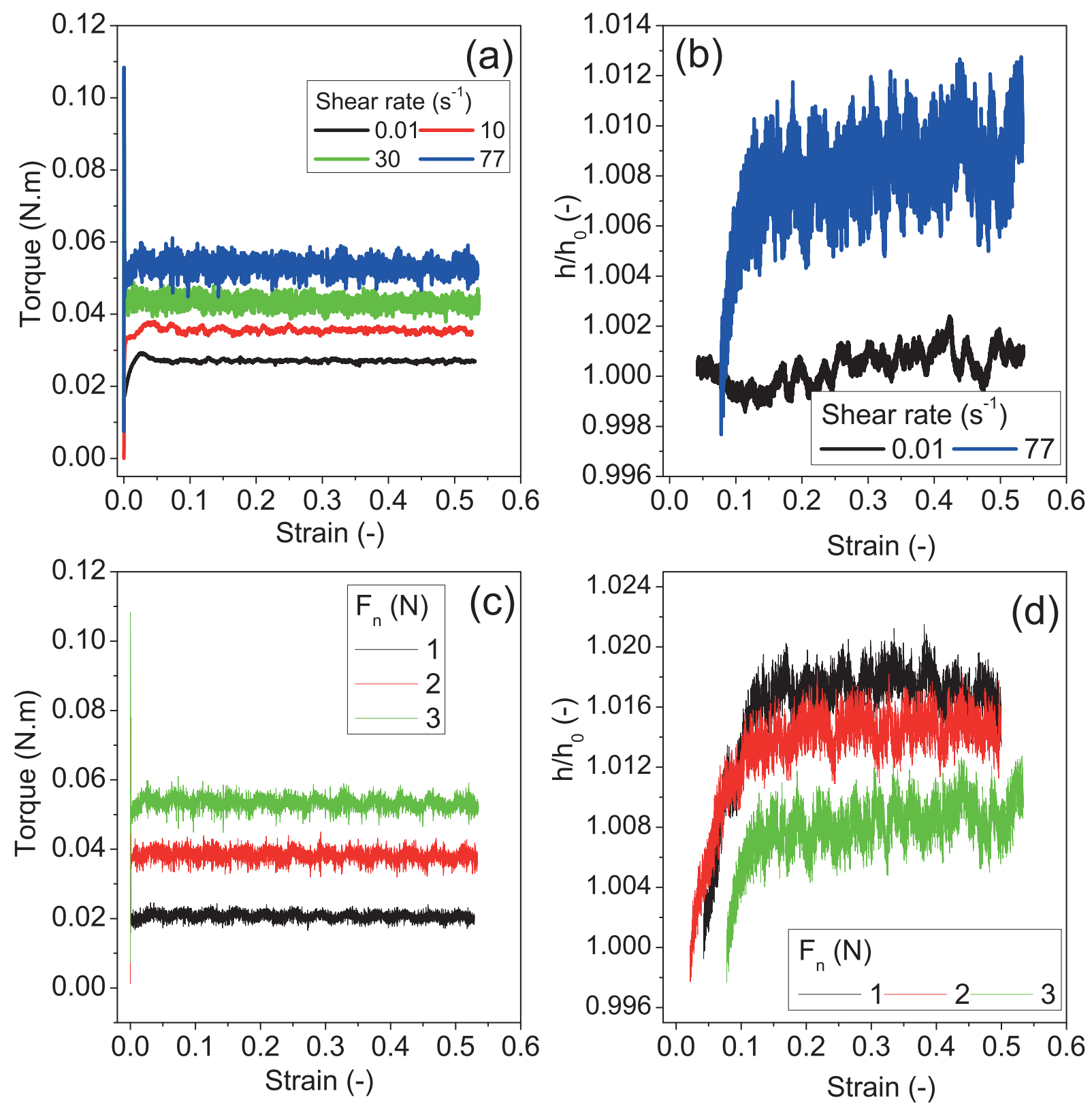

Figure 3: Evolution as a function of the strain at $3 \mathrm{~N}$ imposed normal force under different applied shear rates of: (a) the driving torque and (b) the rescaled gap size (only 2 curves are shown for clarity). Evolution as a function of the strain at $77 \mathrm{~s}^{-1}$ imposed shear rate under different applied normal forces of: (c) the driving torque and (d) the gap size rescaled by its initial value before shearing.

Upon increasing the imposed shear rate, an overshoot occurs: its amplitude increases with increasing the shear rate. In steady state, a rate dependence of the torque is observed (Figure 3a). Moreover, increasing the imposed shear rate causes an increase of the gap size (Figure 3b) allowing to quantify the dynamic dilatancy of the granular material. Notice that, with 
303 increasing the applied shear rate, large fluctuations of the driving torque and also of the gap 304 size evolution occur in steady state flows.

305 Similarly, in experiments in which different normal forces are imposed at a given shear rate 306 (Figure 3c, d), the steady torque is observed to increase while the steady solid fraction (steady 307 gap) decreases when the normal force is increased.

308 Once the above described experiments are combined, we can obtain the constitutive laws of 309 the dry granular material i.e. the dependence of the steady solid fraction $\phi$ and the ratio 310 between shear and normal stresses $\tau / \sigma$ variation on shear rate. Indeed, from macroscopic 311 quantities $T, \Omega, F_{N}$ and $h$, the shear stress $\tau$, the normal stress $\sigma$, shear rate $\dot{\gamma}$, and the 312 solid fraction $\phi$ can be computed.

314 In the annular plate-cup shear geometry, the driving torque is converted into shear stress using 315 the equation:

$$
T=\int_{R_{i}}^{R_{O}} 2 \pi \cdot \tau \cdot r^{2} d r
$$

where $\tau$ is the shear stress, and $R_{i}$ and $R_{o}$ are inner and outer radii of the annular trough.

318 If one neglects the radial velocity gradient (Cleaver et al. 2000; Coste 2004) into the annular 319 trough, the shear stress is quasi-independent of the radial position $r$ and thus, integrating Eq. 320 (4) yields the shear stress as:

$$
\tau=3 T / 2 \pi\left(R_{o}^{3}-R_{i}^{3}\right)
$$

322 Eq. (5) holds because the lateral contribution of wall frictions on the stress distribution within 323 the granular media can be neglected. Indeed, for $h=30 d$, this relative contribution can be 324 estimated to be of the order of $\mu_{w} / \mu$ and we will see below that $\mu_{w} / \mu<<1$.

326 The normal stress can be also calculated from the normal force as follow:

$$
\sigma=F_{N} / \pi\left(R_{o}^{2}-R_{i}^{2}\right)
$$

328 Notice that for $h=10 d$, the imposed normal stress is larger than the hydrostatic pressure $329 \rho g$ once $F_{N}$ is larger than $0.25 N$, meaning that gravity may be neglected for the range of 330 imposed normal forces. 
332 Besides, assuming that the velocity gradient is approximately uniform over the depth and 333 width of the annular trough and a no-slip condition exists at the rough upper and lower 334 shearing walls, one can estimate the mean shear rate as:

$$
\dot{\gamma}=\Omega\left(R_{o}+R_{i}\right) / 2 h
$$

336 And the mean shear strain is given by:

$$
\gamma=\theta\left(R_{o}+R_{i}\right) / 2 h
$$

338 where $\theta$ is the angular displacement.

339 With this analysis, one can plot the shear stress in the steady state as a function of the normal 340 stress as in Figure 4a. The first observation is that a linear relationship between the shear and 341 normal stresses is seen for all imposed shear rates, with a slope that increases from 0.265 to 3420.6 with increasing the shear rate. If an internal friction coefficient $\mu$ is defined as the ratio 343 between shear and normal stresses, we evidence here that $\mu$ is rate dependent: it increases 344 with $\dot{\gamma}$.
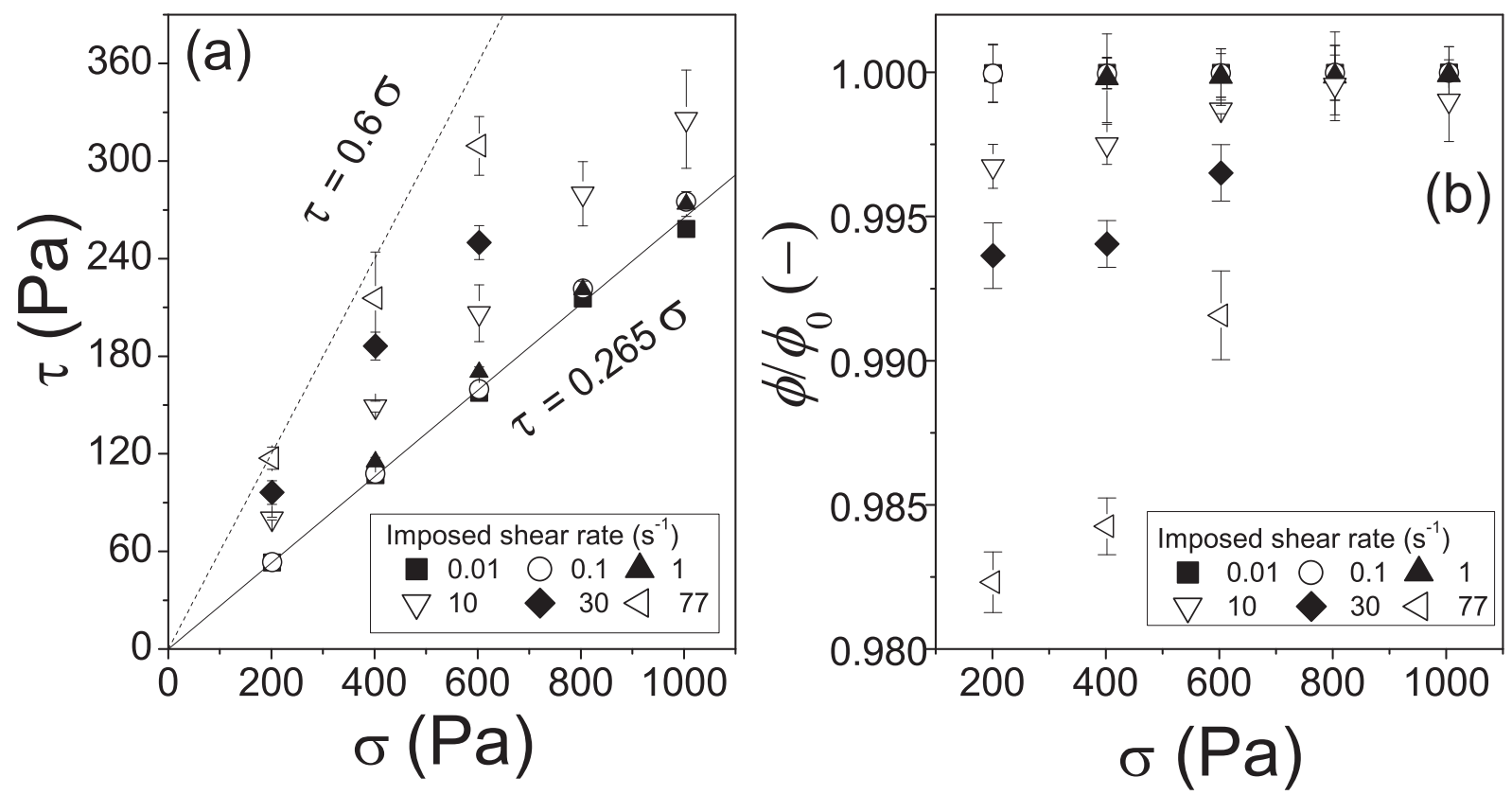

Figure 4: Plot of the shear stress (a) and of the solid fraction rescaled by its initial value before shearing (b) as a function of the normal stress. The stress and the gap are measured in 348 the steady state, for different imposed shear rate. The error bars come from three experiment 349 runs.

351 The second observation is that, for all imposed shear rates, the steady value of the solid 352 fraction decreases when one decreases the normal stress (Figure 4b). Indeed, since the grains 
353

354

355

356

357

358

359

360

361

362

363

364

365

366

367

368

369

370

371

372 Figure 5a shows how $\mu$ vary throughout the flow regimes since $I$ characterizes the local

373 "rapidity" of the flow. All the data obtained for different sets of shear rate and normal force 374

cannot escape from the cell, one can measure unambiguously the solid fraction from the gap variation as:

$$
\phi=m / \pi \rho h\left(R_{o}^{2}-R_{i}^{2}\right)
$$

in which $m$ represents the mass of grains. Thus $h_{0} / h=\phi / \phi_{0}$ will simply reflect the impact of shear and confinement on dilatancy.

In order to analyse the results in term of $\mu(I)$ - rheology, one has to define an inertial number such as:

in which $\sigma$ is the normal stress in the steady state regime. It varies between $10^{-7}$ and 0.1 in the range of applied normal force and shear rate. This corresponds to the usual range of quasistatic to dense flow regimes. It should be noted that our annular shear geometry does not allow higher values of $I$ to be studied.
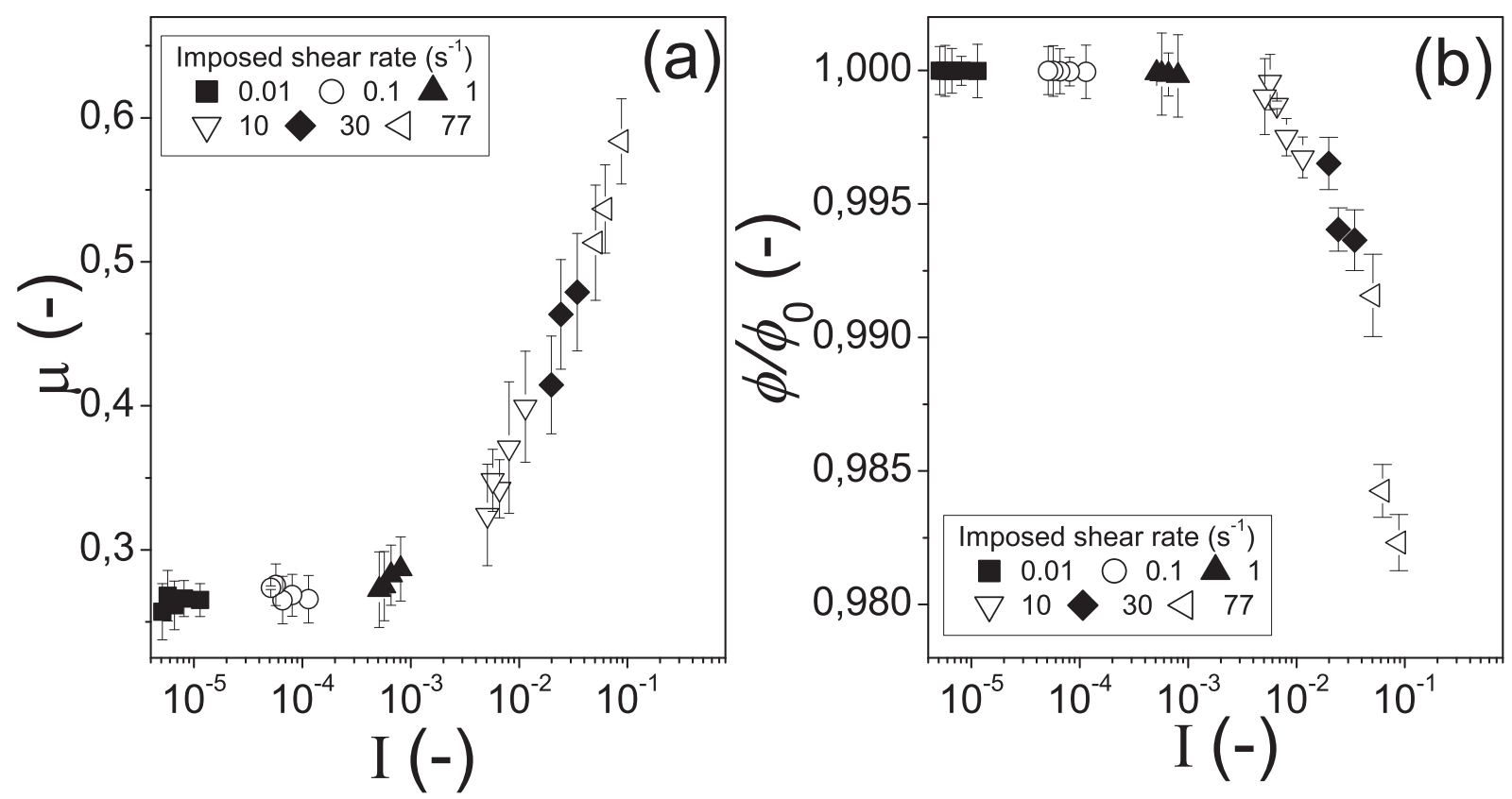

Figure 5: Constitutive law for different sets of mean shear rates and imposed normal forces (a) 'friction law' i.e. effective internal friction coefficient as a function of inertial number; (b) 'dynamic dilatancy law' i.e. solid fraction as a function of inertial number. The error bars come from three experiment runs.

collapse on a single curve $\mu=\tau / \sigma$ vs. $I$. For low inertial number, the internal friction 
coefficient tends to a finite value $\mu_{s} \approx 0.265$ and increases with increasing $I$. At the same time, the solid fraction variation $\phi / \phi_{0}$ with the inertial number $I$ is shown in Figure 5b. Once again, all the data collapse on a single curve. At low $I, \phi / \phi_{0}$ is quasi-constant: this is the quasi-static regime. When $I$ increases, the inertia starts influencing the flow and the system becomes rate dependent: the ratio $\phi / \phi_{0}$ decreases; this regime corresponds to the dense flow in which the granular material dilates.

Moreover, in order to check the robustness of our results, we have varied the initial size of the gap. Here, with our annular shear geometry, the same experiments discussed above are made with different gap sizes from $6 d$ to $22 d$ and different sets of imposed shear rate and normal force (Table 1).

\begin{tabular}{|c|c|c|}
\hline Shear rate $\left(\mathrm{s}^{-1}\right)$ & Normal force $\mathbf{F}_{\mathbf{N}}(\mathrm{N})$ & Gap $\left(\boldsymbol{h}_{0} / \boldsymbol{d}\right)$ \\
\hline 0.01 & $1-2-3-4-5$ & $6-8-15$ \\
\hline 0.1 & $1-2-3-4-5$ & $6-8-10$ \\
\hline 1 & $1-2-3-4-5$ & $6-10-15$ \\
\hline 10 & $1-2-3$ & $6-15$ \\
\hline 30 & $1-2-3$ & $6-15-22$ \\
\hline 77 & $1-2-3$ & $6-15-22$ \\
\hline
\end{tabular}

386 387

Table 1: Values of the imposed shear rate and normal force for each gap size.

It is shown in Figure 6 that changing the gap does not significantly affect these results. This suggests a total absence of shear localization at high inertial number. However, at small inertial number, the resolution of our measurements is not sufficient to dismiss the possibility that shear localization arises. It was indeed shown that in confined annular flow at small shear velocities and high confining pressures - small $I$-, the shear may be not homogeneous and solid and fluid phases coexist (Aharonov \& Sparks 2002; Jalali et al. 2002).

We now compare these experimental results with existing models such as those of Jop et al. Eq. (1) and Hatano Eq. (3) on dry granular flows in terms of quasi-static and dense flow behaviours. Concerning the 'friction law', our experimental data include points in range of $I$ from $10^{-7}$ to 0.1 which covers quasi-static and dense flow regimes. Our measurements then show that, in this range of $I$, both models can describe our data. Using a classical least squares method, the fit of the data gives: $I_{0} \approx 0.032 \pm 0.003, \mu_{s} \approx 0.271 \pm 0.002$ and 
$\mu_{2} \approx 0.68 \pm 0.02$ for the 'Jop et al. model'; $\mu_{s} \approx 0.259 \pm 0.002, \quad a \approx 1.12 \pm 0.05$ and

$402 n \approx 0.49 \pm 0.02$ for the 'Hatano model'. In Figure $6 \mathrm{~b}$, we show the 'dynamic dilatancy law', 403 i.e., the variation of the solid fraction $\phi$ as a function of the inertial number $I$. We observe 404 that $\phi$ decreases linearly with $I$, starting from a maximum value $\phi_{c}$ in the quasi-static regime 405 where the granular material is very dense, close to the maximum solid fraction. The 'dynamic 406 dilatancy law' is thus given as: $\phi / \phi_{c}=1-\left(1-\phi_{m} / \phi_{c}\right) I$ with $\phi_{c} \approx \phi_{0} \approx 0.625$ and $\phi_{m} \approx 0.495 \pm 0.002$ in agreement with (Pouliquen et al. 2006; Jop et al. 2005).
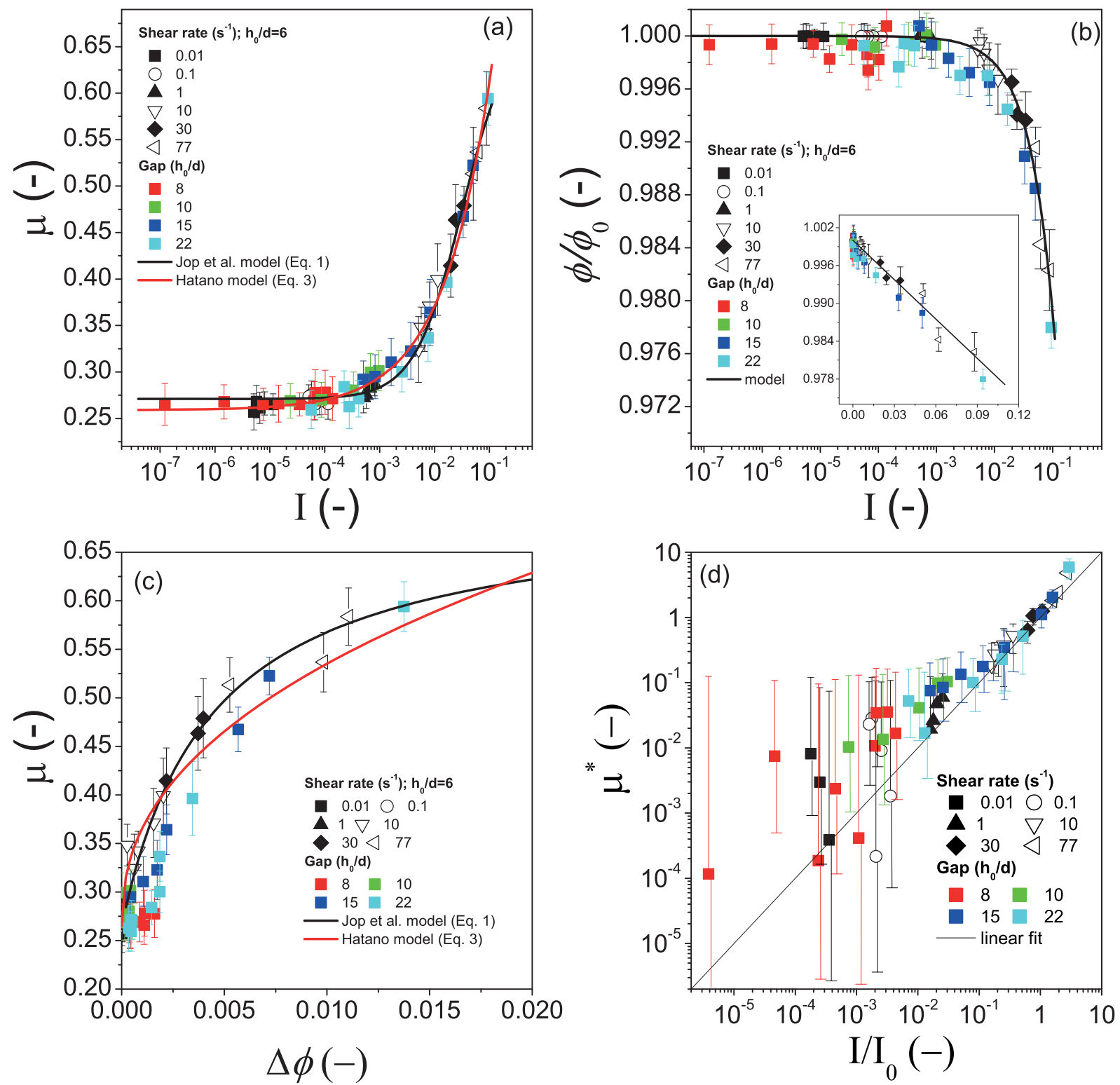

409 Figure 6: Constitutive law for different sets of mean shear rates and imposed normal forces, 410 and different gap sizes (Table 1): (a) 'friction law' i.e. internal friction coefficient as a 411 function of inertial number; (b) 'dynamic dilatancy law' i.e. solid fraction as a function of 412 inertial number; inset: the same data in linear scale. The solid line is $\phi / \phi_{c}=1-\left(1-\phi_{m} / \phi_{c}\right) I$ 
413 with $\phi_{c} \approx \phi_{0} \approx 0.625$ and $\phi_{m} \approx 0.495$; (c) Internal friction coefficient vs. the reduced solid

414 fraction ( $\left.\Delta \phi=\phi_{c}-\phi\right)$; (d) Reduced internal friction coefficient as a function of the reduced 415 inertial number. The line is $\mu^{*}=I / I_{0}$. For each gap, the imposed shear rate varies from 0.01 416 to $77 s^{-1}$ depending of the imposed normal force in order to obtain either a low $I$ (small $\dot{\gamma}$ 417 and/or large $F_{N}$ ) or a high $I$ (large $\dot{\gamma}$ and/or small $F_{N}$ ). The error bars come from three 418 experiment runs.

419

Combining the 'dynamic dilatancy' and the 'friction' laws, these data show that the internal friction coefficient $\mu$ strongly depends on the solid fraction: it decreases towards $\mu_{\mathrm{s}}$ when $\phi$ tends to the maximum solid fraction (as shown on Figure 6c in which $\Delta \phi=\phi_{0}-\phi$ is the reduced solid fraction). Moreover, following Staron and co-workers (Staron et al. 2010), we defined a reduced internal friction coefficient $\mu^{*}$ as:

$$
\mu^{*}=\left(\mu-\mu_{s}\right) /\left(\mu_{2}-\mu\right)
$$

We plot in the main panel of Figure 6d the resulting $\mu^{*}$ vs. $I / I_{0}$ data points. It holds remarkably well with a prefactor of unity. Satisfying the 'Jop et al. model' implies indeed that $\mu^{*}=I / I_{0}$ which is almost the case for our data except for small values of $I\left(I<I_{0}=0.03\right)$ wherein deviations seem to take place. The relationship between $\mu^{*}$ and the reduced inertial number is not clear. These deviations, observed in the quasi-static regime, were also mentioned in recent studies which indicate that this rheology (the 'Jop et al. model') may be not sufficient to describe the complex phenomena occurring at the flow threshold such as intermittent flows (Mills et al. 2008), and is maybe strictly valid only for relatively large inertial numbers (e.g., $I>0.02$ as noted by Staron et al. 2010 and $I>0.005$ by Gaume et al. 2011). The reason for that is not clear yet but one possible explanation is shear localization that arises most often in confined annular flow at small imposed shear and high pressure (small inertial number I) (Aharonov \& Sparks 2002; Jalali et al. 2002; Koval 2009). Such behavior of granular materials has not yet been fully understood and no consistent and general formalism can predict it successfully (Kamrin 2012). In contrast to discrete numerical simulations (Wang et al. 2012) and theoretical studies (Jenkins \& Richman 1985; Richman \&

441 Chou 1988; Jenkins 1992), the study of shear localization structure with experimental 442 methods is rather difficult. The visualization of the granular interface is usually limited to the 
to study the granular rheology (velocity and solid fraction profiles) inside the granular system (Moucheront et al. 2010); this tool may thus be a great help in understanding the behaviour at low I. A change in the roughness of a boundary bottom might be used to modify the flow properties such as the wall slip velocity (Shojaaee et al. 2012).

\section{Conclusion}

We have developed a rheometrical method in order to study dense granular flows with a rheometer under imposed confining normal stress and applied shear rate. From the steady state measurement of the torque and the gap, the internal friction coefficient, the solid fraction and the inertial number $I$ are measured. For low $I$, the flow goes to the quasi-static limit and the internal friction coefficient and the solid fraction profiles are independent of $I$. Upon increasing $I$, dilation occurs and the solid fraction decreases linearly when $I$ increases while the friction coefficient increases. The observed variations are in good agreement with previous observations of the literature. As a consequence, we bring evidence that rheometric measurements can be relevant to describe dry granular flows. However, additional experimental work should be carried out in order to measure the dependence of the boundary layer constitutive law on the state of the bulk material, so as to be able to describe properly the rheology when approaching the quasi-static limit.

\section{Acknowledgments}

The authors thank P. Mills for useful discussions, a critical reading of the manuscript and many useful remarks.

\section{References}

E. Aharonov \& D. Sparks, Shear profiles and localization in simulations of granular materials, Physical Review E, 65:051302, 2002.

B. Andreotti, Y. Forterre and O. Pouliquen, Granular Media: Between Fluid and Solid, Cambridge University Press, 2013.

C. Ancey, P. Coussot, P. Evesque, A theoretical framework for granular suspensions in a steady simple shear flow, J. Rheol. 43 (6), 1673-1699, 1999.

I. S. Aranson, L. S. Tsimring, F. Malloggi, and E. Clement, Nonlocal rheological properties of granular flows near a jamming limit, Phys. Rev. E 78, 031303, 2008. 
E. Azéma and F. Radjai, Internal Structure of Inertial Granular Flows, Phys. Rev. Lett. 112, 078001, 2014.

R. A. Bagnold, Experiments on a gravity free dispersion of large solid spheres in a newtonian fluid under shear, Proc. Roy. Soc. London Ser. A, 225, 1954.

O. Baran and L. Kondic, On velocity profiles and stresses in sheared and vibrated granular systems under variable gravity, Phys. Fluids 18, 121509, 2006.

M. Becker and H. Lippmann, Plane plastic flow of granular model material. Experimental setup and results, Archiv of Mechanics, 29(6):829-846, 1977.

F. Boyer, E. Guazzelli, O. Pouliquen, Unifying suspension and granular rheology, Phys. Rev. Lett. 107, 188301, 2011.

J.-F. Camenen, Y. Descantes and P. Richard, Effect of confinement on dense packings of rigid frictionless spheres and polyhedral, Phys. Rev. E, 86, 061317, 2012.

J.F. Carr and D.M. Walker, An annular shear cell for granular materials, Powder Tech 1(6): 369-373, 1968.

S. Chialvo, J. Sun, and S. Sundaresan, Bridging the rheology of granular flows in three regimes, Phys. Rev. E 85, 021305, 2012.

J.A.S. Cleaver, R.M. Nedderman and R.B. Thorpe, Accounting for granular material dilation during the operation of an annular shear cell, Adv. Powder Tech. 11, 4, 385-399, 2000.

C. Coste, Shearing of a confined granular layer: Tangential stress and dilatancy, Phys. Rev. E, 70, $051302,2004$.

F. da Cruz, S. Emam, M. Prochnow, J. Roux, and F. Chevoir, Rheophysics of dense granular materials: Discrete simulation of plane shear flows, Phys. Rev. E., 72:021309, 2005.

J. Duran, Sands, powders and grains, Springer-Verlag, New-York, 2000.

A. N. Edwards \& J. M. N. T. Gray, Erosion-deposition waves in shallow granular freesurface flows, J. Fluid Mech., vol. 762, pp. 35-67, 2015.

D. Fenistein and M. van Hecke, Wide shear zones in granular bulk flow. Nature, 425:256, 2003.

503 D. Fenistein, J.W. van de Meent, and M. van Hecke, Universal and wide shear zones in granular bulk flow, Phys. Rev. Lett. 92, 094301, 2004.

Y. Forterre and O. Pouliquen, Flow of dense granular media, Annual Reviews of Fluid Mechanics 40, 1-24, 2008.

GDR Midi. On dense granular flows. Euro. Phys. Journ. E., 14:341-365, 2004.

J. Gaume, G. Chambon, and M. Naaim, Quasistatic to inertial transition in granular materials and the role of fluctuations, Phys. Rev. E 84, 051304, 2011. 
I. Goldhirsch, Rapid granular flows, Annual Reviews of Fluid Mechanics 35, 267-293, 2003.

J. M. N. T. Gray \& A. N. Edwards, A depth-averaged $\mu(I)$-rheology for shallow granular free-surface flows, J. Fluid Mech., vol. 755, pp. 503-534, 2014.

\section{3}

514

515

516

517

518

519

520

521

522

523

524

525

526

527

528

529

530

531

532

533

534

535

536

537

538

539

540

541

542

T. Hatano, Power-law friction in closely packed granular materials, Phys. Rev. E, 75, 060301 (R), 2007

I. Iordanoff and M. M. Khonsari, Granular lubrication: toward an understanding between kinetic and fluid regime, ASME J. Tribol., 126:137-145, 2004.

P. Jalali, W. Polashenski Jr., T. Tynjälä, and P. Zamankhan, Particle interactions in a dense monosized granular flow, Physica D, 162:188-207, 2002.

A.W. Jenike, Storage and flow of solids, Bulletin No. 123, Utah Engineering Station, Salt Lake City, UT., 1964

J. T. Jenkins and S. B. Savage, A theory for the rapid flow of identical smooth nearly elastic spherical particles, J. Fluid Mech. 130, 187, 1983.

J. T. Jenkins and M. W. Richman, Kinetic theory for plane flows of a dense gas of identical, rough, inelastic, circular disks, Physics of Fluids, 28(12):3485, 1985.

J. T. Jenkins, Boundary conditions for rapid granular flows: Flat, frictional walls. J. Appl. Mech., 59:120, 1992.

P. Jop, Y. Forterre, and O. Pouliquen, Crucial role of sidewalls in granular surface flows: consequences for the rheology, J. Fluid Mech. 541, 167, 2005.

P. Jop, Y. Forterre and O. Pouliquen, A constitutive law for dense granular flows, Nature 441,727, 2006.

K. Kamrin and G. Koval, Nonlocal Constitutive Relation for Steady Granular Flow, Phys. Rev. Lett. 108, 178301, 2012.

G. Koval, J.-N. Roux, A. Corfdir, and F. Chevoir, Annular shear of cohesionless granular materials: From the inertial to quasistatic regime, Physical Review E, 79(2):021306, 2009.

G. Lois, A. Lemaitre, and J. M. Carlson, Numerical tests of constitutive laws for dense granular flows, Phys. Rev. E., 72, 051303, 2005.

S. Luding, The effect of friction on wide shear bands, Part. Sci. Technol., 26-33, 2008.

C.W. Macosko, Rheology: Principles, Measurements and Applications; Wiley VCH: New York, 1994.

P. Mills, P. Rognon and F. Chevoir, Rheology and structure of granular materials near the jamming transition, Euro. Phys. Lett., 81, 64005, 2008. 
P. Moucheront, F. Bertrand, G. Koval, L. Tocquer, S. Rodts, J.-N. Roux, A. Corfdir, and F. Chevoir, MRI investigation of granular interface rheology using a new cylinder shear apparatus, Magnetic Resonance Imaging, 28(6):910-918, 2010.

R.M. Nedderman, Statics and kinematics of granular materials. Cambridge University Press, Cambridge, 1992.

G. Ovarlez, C. Fond and E. Clément, Overshoot effect in the Janssen granular column: a crucial test for granular mechanics, Phys. Rev. E 67, 060302(R), 2003.

P-E. Peyneau and J-N. Roux, Frictionless bead packs have macroscopic friction, but no dilatancy, Phys. Rev. E 78:011307, 2008

O. Pouliquen, C. Cassar, P. Jop, Y. Forterre and M. Nicolas, Flow of dense granular material: towards simple constitutive laws, J. Statist. Mech., 7, P07020, 2006.

O. Pouliquen and Y. Forterre, Friction law for dense granular flows: application to the motion of a mass down a rough inclined plane, J. Fluid Mech., 453, 133-151, 2002.

M. W. Richman and C. S. Chou, Boundary effects on granular shear flows of smooth disks, Journal of Applied Mathematics and Physics (ZAMP), 39(6):885-901, 1988.

J. -N. Roux and G. Combe, Rhéologie quasi-statique et origines de la déformation, Comptes Rendus Physique 3, 131, 2002.

A. de Ryck, R. Ansart and J. A. Dodds, Granular flows down inclined channels with a strainrate dependent friction coefficient, Part I: Non-cohesive materials, Granular Matter 10, 353, 2008.

S. B. Savage and M. Sayed, Stresses developed by dry cohesionless granular materials sheared in an annular shear cell, J. Fluid. Mech., 142, 391-430, 1984.

S. B. Savage \& K. Hutter, The motion of a finite mass of granular material down a rough incline, J. Fluid Mech., 199, 177-215, 1989.

A. Schoefield and P. Wroth, Critical State Soil Mechanics, McGraw-Hill, 1968.

Z. Shojaaee, J.-N. Roux, F. Chevoir, D. E. Wolf, Shear flow of dense granular materials near smooth walls. I. Shear localization and constitutive laws in the boundary region, Phys. Rev. E 86 (1), 011301, 2012.

D. Schulze, Flowability and time consolidation measurements using a ring shear tester. Powder Handl Proc. 8:221-226, 1996.

J. Schwedes, Review on testers for measuring flow properties of bulk solids, Granular Matter 5, 1, 2003.

L.E. Silbert, J. W. Landry \& G. S. Grest, Granular flow down a rough inclined plane: transition between thin and thick piles, Phys. Fluids 15, 1-10, 2003. 
L. E. Silbert, D. Ertas, G. S. Grest, T. C. Halsey, D. Levine, and S. J. Plimpton, Granular flow down an inclined plane: Bagnold scaling and rheology, Phys. Rev. E., 64:051302,

579 2001.

580

L. Staron, P.-Y. Lagrée, C. Josserand, and D. Lhuillier, Flow and jamming of a twodimensional granular bed: Toward a nonlocal rheology? Phys. Fluids 22, 113303,

582 2010.

583

S. Torquato, T. M. Truskett, and P. G. Debenedetti, Is Random Close Packing of Spheres Well

584 Defined? Phys. Rev. Lett. 84, 2064, 2000.

585

A. Tripathi and D. V. Khakhar, Rheology of binary granular mixtures in the dense flow 586 regime, Phys. Fluids 23, 113302, 2011.

X. Wang, H. P. Zhu, and A. B. Yu, Flow properties of particles in a model annular shear cell, Phys. Fluids 24, 053301, 2012.

589 C.P. Wroth, Soil behaviour during shear - existence of critical void ratios, Engineering 186, 409, 1958. 